Research Article

\title{
A Note on $\mathscr{L} \mathscr{P}$-Sasakian Manifolds with Almost Quasi-Yamabe Solitons
}

\author{
Sunil Kumar Yadav $\mathbb{D}^{1},{ }^{1}$ D. L. Suthar $\mathbb{D},^{2}$ and Biniyam Shimelis $\mathbb{D}^{2}$ \\ ${ }^{1}$ Department of Applied Science and Humanities, United College of Engineering \& Research, \\ A-31, UPSIDC Industrial Area, Naini, Prayagraj-211010, Uttar Prasdesh, India \\ ${ }^{2}$ Department of Mathematics, Wollo University, P. O. Box 1145, Dessie, Ethiopia \\ Correspondence should be addressed to Biniyam Shimelis; biniyam.shimelis@wu.edu.et
}

Received 27 August 2021; Revised 16 October 2021; Accepted 9 November 2021; Published 10 December 2021

Academic Editor: Ram Jiwari

Copyright (C) 2021 Sunil Kumar Yadav et al. This is an open access article distributed under the Creative Commons Attribution License, which permits unrestricted use, distribution, and reproduction in any medium, provided the original work is properly cited.

We categorize almost quasi-Yamabe solitons on $\mathscr{L} \mathscr{P}$-Sasakian manifolds and their $\mathscr{C} \mathscr{R}$-submanifolds whose potential vector field is torse-forming, admitting a generalized symmetric metric connection of type $(\alpha, \beta)$. Finally, a nontrivial example is provided to confirm some of our results.

\section{Introduction}

Yamabe solitons (YS) are ideas that generate self-similar Yamabe flow (YF) solutions [1]:

$$
\frac{\partial}{\partial \check{t}} \ddot{g}(\check{t})=-\widehat{\delta}(\check{t}) \ddot{g}(\check{t}) \text {. }
$$

Di Cerbo and Disconzi were the first to notice them in [2]. Chen and Deshmukh proposed the concept of quasiYamabe soliton (QYS) in [3], which we will explore in this study for a more broader situation when the constants are functions.

Definition 1. An almost quasi-Yamabe solitons (AQYS) on Remannian manifold $\left(\Theta^{n}, \ddot{g}\right)$ is a set of data $(\ddot{g}, \mathscr{K}, \mu, \widehat{\sigma})$ that fulfill the following equation:

$$
\frac{1}{2} \mathfrak{Q}_{\mathscr{K}} \ddot{g}=(\widehat{\delta}-\widehat{\sigma}) \ddot{g}+\mu \mathscr{K}^{\diamond} \otimes \mathscr{K}^{\diamond},
$$

where $\mathfrak{Q}_{\mathscr{K}}$ is operator of the Lie derivative in view of $\mathscr{K}, \widehat{\sigma}$ and $\mu$ are smooth functions on $\left(\Theta^{n}, \ddot{g}\right), \mathscr{K}^{\diamond}$ is the 1-form of $\mathscr{K}$, and $\widehat{\delta}$ is the scalar curvature. If $\widehat{\sigma}<0, \widehat{\sigma}=0$, or $\widehat{\sigma}>0$, we call an AQYS shrinking, stable, or growing, respectively, using Yamabe solitons nomenclature.

If $\mathscr{K}=\nabla \widetilde{f}$ is a gradient type, (2) yields

$$
\operatorname{Hess}(\widehat{f})=(\widehat{\delta}-\widehat{\sigma}) \ddot{g}+\mu \mathrm{d} \hat{f} \otimes \mathrm{d} \widehat{f} .
$$

It is nothing more than a generalized quasi-Yamabe gradient soliton (GQYGS) (see [4, 5]). Several authors have thoroughly investigated AQYS and QYGS in [6-13].

Definition 2. A vector field $\mathscr{K}$ on $\left(\Theta^{\hat{n}}, \ddot{g}\right)$ meets the following conditions and is known as a torse-forming vector field [14]:

$$
\nabla_{\mathscr{P}^{*}} \mathscr{K}=\psi \mathscr{P}^{*}+\pi \mathscr{P}^{*} \mathscr{K}, \quad \forall \mathscr{P}^{*} \in \chi(\Theta),
$$

where $\pi$ is a 1 -form and $\psi$ is in $C^{\infty}(\Theta)$.

We classify such vector field as

(i) It is concircular if the 1 -form $\pi$ vanishes identically [15]

(ii) For concurrent, $\psi=1$ and $\pi=0[16]$

(iii) It is recurrent if $\psi=0$

(iv) Parallel if $\psi=\pi=0$

If the vector field $\mathscr{K}$ fulfills (4) with $\pi(\mathscr{K})=0$, it called as torqued vector field [17].

The content of the paper is as follows. After the opening remark, Section 2 contains the fundamental result of an 
$\mathscr{L} \mathscr{P}$-Sasakian manifold. We discuss the concept of a generalized symmetric metric connection- $(\alpha, \beta)(\operatorname{GSMC}-(\alpha, \beta))$ in Section 3. With regard to GSMC- $(\alpha, \beta)$, Section 4 is devoted to $\mathscr{C} \mathscr{R}$-submanifolds of an $\mathscr{L} \mathscr{P}$-Sasakian manifold. With respect to such a connection, we examine QYS in view of a torse-forming vector field on an $\mathscr{L} \mathscr{P}$-Sasakian manifold in Section 5. The study of QYS with a torse-forming vector field on $\mathscr{C} \mathscr{R}$-submanifolds of an $\mathscr{L} \mathscr{P}$-Sasakian manifolds is also covered in Section 6. Finally, in Section 7, we look at AQYGS with a torse-forming vector field by considering the tangential $\mathscr{K}^{t}$ and normal $\mathscr{K}^{n}$ components of such a vector field on $\mathscr{C} \mathscr{R}$-submanifolds.

\section{LP-Sasakian Manifolds}

If a $(1,1)$ tensor field $\phi_{*}$, a contravariant vector field $\widehat{\zeta}$, a 1 form $\eta_{*}$, and the Lorentzian metric $\ddot{g}$ are admitted to a differentiable manifold $\Theta^{n}$; it is termed as an $\mathscr{L} \mathscr{P}$-Sasakian manifold $\Theta^{\widehat{n}}\left(\phi_{*}, \widehat{\zeta}, \eta_{*}, \ddot{g}\right)$ (see $\left.[18,19]\right)$; then,

$$
\begin{aligned}
\eta_{*}(\widehat{\zeta}) & =-1, \\
\phi_{*}^{2}\left(\mathscr{P}^{*}\right) & =\mathscr{P}^{*}+\eta_{*}\left(\mathscr{P}^{*}\right) \hat{\zeta}, \\
\ddot{g}\left(\phi_{*} \mathscr{P}^{*}, \phi_{*} \mathscr{Q}^{*}\right) & =\ddot{g}\left(\mathscr{P}^{*}, \mathscr{Q}^{*}\right)+\eta_{*}\left(\mathscr{P}^{*}\right) \eta_{*}\left(\mathscr{Q}^{*}\right), \\
\ddot{g}\left(\mathscr{P}^{*}, \widehat{\zeta}\right) & =\eta_{*}\left(\mathscr{P}^{*}\right), \\
\nabla_{\mathscr{P}^{*}} \widehat{\zeta} & =\phi_{*} \mathscr{P}^{*}, \\
\left(\nabla_{\mathscr{P}^{*}} \phi_{*}\right)\left(\widehat{Q}^{*}\right)= & \ddot{g}\left(\mathscr{P}^{*}, \mathscr{Q}^{*}\right) \widehat{\zeta}+\eta_{*}\left(\mathscr{Q}^{*}\right) \mathscr{P}^{*} \\
& +2 \eta_{*}\left(\mathscr{P}^{*}\right) \eta_{*}\left(\mathscr{Q}^{*}\right),
\end{aligned}
$$

where $\nabla$ be the Levi-Civita connection along the metric $\ddot{g}$. In an $\mathscr{L} \mathscr{P}$-Sasakian manifold, we yields

$$
\begin{aligned}
\phi_{*} \widehat{\zeta} & =0, \\
\eta_{*}\left(\phi_{*} \mathscr{P}^{*}\right) & =0, \\
\operatorname{rank}\left(\phi_{*}\right) & =\widehat{n}-1 .
\end{aligned}
$$

If we write

$$
\widehat{\Phi}\left(\mathscr{P}^{*}, Q^{*}\right)=\ddot{g}\left(\phi_{*} \mathscr{P}^{*}, Q^{*}\right), \quad \mathscr{P}^{*}, Q^{*} \in \Theta^{\widehat{n}},
$$

then $\widehat{\Phi}\left(\mathscr{P}^{*}, Q^{*}\right)$ is a symmetric $(0,2)$ tensor field. Now, $\eta_{*}$ is closed on $\Theta^{\widehat{n}}\left(\phi_{*}, \widehat{\zeta}, \eta_{*}, \ddot{g}\right)$ (see $\left.[18,20]\right)$; then,

$$
\left(\nabla_{\mathscr{P}^{*}} \eta\right)\left(\widehat{Q}^{*}\right)=\widehat{\Phi}\left(\mathscr{P}^{*}, \widehat{Q}^{*}\right), \quad \widehat{\Phi}\left(\mathscr{P}^{*}, \widehat{\zeta}\right)=0 .
$$

In an $\mathscr{L} \mathscr{P}$-Sasakian manifold $\left(\Theta^{\hat{n}}, \ddot{g}\right)$, the following relationships are maintained (see $[20,21])$ :

$$
\begin{aligned}
\ddot{g}\left(\mathscr{R}\left(\mathscr{P}^{*}, \mathscr{Q}^{*}\right) \mathscr{Z}^{*}, \widehat{\zeta}\right) & =\ddot{g}\left(\mathscr{Q}^{*}, \mathscr{Z}^{*}\right) \eta_{*}\left(\mathscr{P}^{*}\right)-\ddot{g}\left(\mathscr{P}^{*}, \mathscr{Z}^{*}\right) \eta_{*}(Q), \\
\ddot{g}\left(\mathscr{R}\left(\widehat{\zeta}, \mathscr{P}^{*}\right) \mathscr{Q}^{*}\right) & =\ddot{g}\left(\mathscr{P}^{*}, \mathscr{Q}^{*}\right) \widehat{\zeta}-\eta_{*}\left(\mathscr{Q}^{*}\right) \mathscr{P}^{*}, \\
\mathscr{R}\left(\mathscr{P}^{*}, Q^{*}\right) \widehat{\zeta} & =\eta_{*}\left(\mathscr{Q}^{*}\right) \mathscr{P}^{*}-\eta_{*}\left(\mathscr{P}^{*}\right) \mathscr{Q}^{*}, \\
\mathscr{R}\left(\widehat{\zeta}, \mathscr{P}^{*}\right) \widehat{\zeta} & =\mathscr{P}^{*}+\eta_{*}\left(\mathscr{P}^{*}\right) \widehat{\zeta}, \\
\mathcal{S}\left(\mathscr{P}^{*}, \widehat{\zeta}\right) & =(\widehat{n}-1) \eta_{*}\left(\mathscr{P}^{*}\right), \\
\mathcal{S}\left(\phi_{*} \mathscr{P}^{*}, \phi_{*} \mathscr{Q}^{*}\right) & =\mathcal{S}\left(\mathscr{P}^{*}, \mathscr{Q}^{*}\right)+(\widehat{n}-1) \eta_{*}\left(\mathscr{P}^{*}\right) \eta_{*}\left(\mathscr{Q}^{*}\right),
\end{aligned}
$$

for any vector fields $\mathscr{P}^{*}, Q^{*}$, and $\mathscr{Z}^{*}$ on $\Theta^{\widehat{n}}$, where $\mathscr{R}$ and $\mathcal{S}$ are the curvature tensor and Ricci tensor of $\Theta^{\hat{n}}$, simultaneously.

Let $\Theta$ be a submanifold of an $\mathscr{L} \mathscr{P}$-Sasakian manifold. The Gauss and Weingarten formulas are given by

$$
\begin{gathered}
\nabla_{\mathscr{P}^{*}} \mathscr{Q}^{*}=\dot{\nabla} \mathscr{P}^{*} \mathscr{Q}^{*}+h\left(\mathscr{P}^{*}, Q^{*}\right), \quad \forall \mathscr{P}^{*}, \mathscr{Q}^{*} \in \Gamma\left(T^{*} \grave{\Theta}\right), \\
\nabla_{\mathscr{P}^{*}} \mathscr{N}^{*}=-A_{\mathscr{N}^{*}} \mathscr{P}^{*}+\nabla_{\mathscr{P}^{*}}^{\perp} \mathcal{N}^{*}, \quad \forall \mathscr{N}^{*} \in \Gamma\left(T^{* \perp} \grave{\Theta}\right),
\end{gathered}
$$

where $\nabla_{\mathscr{P}^{*}} \mathscr{Q}^{*}$ and $\left\{h\left(\mathscr{P}^{*}, Q^{*}\right), \nabla_{\mathscr{P}^{*}}^{\perp} \mathscr{N}^{*}\right\}$ belong to $\Gamma\left(T^{\prime} \Theta\right)$ and $\Gamma\left(T^{* \perp} \Theta\right)$, respectively.

\section{Generalized Symmetric Metric Connection of Type $(\alpha, \beta)$}

Let $\widetilde{\nabla}$ and $\nabla$ be a linear and Levi-Civita connection on an $\mathscr{L} \mathscr{P}$-Sasakian manifold. Now, we will go through the results that will be used.

Lemma 1 (see [22]). In an $\mathscr{L} \mathscr{P}$-Sasakian manifold $\Theta^{\widehat{n}}\left(\phi_{*}, \widehat{\zeta}, \eta_{*}, \ddot{g}\right)$, the GSMC $\widetilde{\nabla}$ of type $(\alpha, \beta)$ is given by

$$
\begin{aligned}
\tilde{\nabla}_{\mathscr{P}^{*}}{Q^{*}}^{*} & \nabla_{\mathscr{P}^{*}} \mathscr{Q}^{* *}+\alpha\left\{\eta_{*}\left(\mathscr{Q}^{*}\right) \mathscr{P}^{*}-\ddot{g}\left(\mathscr{P}^{*} P^{*}, Q^{*}\right) \widehat{\zeta}\right\} \\
& +\beta\left\{\eta_{*}\left(\mathscr{Q}^{*}\right) \phi_{*} \mathscr{P}^{*}-\ddot{g}\left(\phi_{*} \mathscr{P}^{*}, \mathscr{Q}^{*}\right) \widehat{\zeta}\right\},
\end{aligned}
$$

for all $\mathscr{P}^{*}$ and $\mathscr{Q}^{*}$ on $\Theta^{\widehat{n}}$.

Lemma 2 (see [22]). The following relations hold on an $\mathscr{L} \mathscr{P}$-Sasakian manifold in light of GSMC- $(\alpha, \beta)$ :

$$
\begin{aligned}
& \left(\widetilde{\nabla}_{\mathscr{P}^{*}} \phi_{*}\right)\left(\mathscr{Q}^{*}\right)=\left[(1-\beta)\left\{\ddot{g}\left(\mathscr{P}^{*}, \mathscr{Q}^{*}\right)+2 \eta_{*}\left(\mathscr{P}^{*}\right) \eta_{*}\left(\mathscr{Q}^{*}\right)\right\}\right. \\
& \left.-\alpha \widehat{\Phi}\left(\mathscr{P}^{*}, Q^{*}\right)\right] \widehat{\zeta} \\
& +(1-\beta) \eta_{*}\left(\mathscr{Q}^{*}\right) \mathscr{P}^{*}-\alpha \eta_{*}\left(\mathscr{Q}^{*}\right) \phi_{*} \mathscr{P}^{*}, \\
& \tilde{\nabla}_{\mathscr{P}^{*}} \widehat{\zeta}=(1-\beta) \phi_{*} \mathscr{P}^{*}-\alpha \mathscr{P}^{*}-\alpha \eta_{*}\left(\mathscr{P}^{*}\right) \widehat{\zeta}, \\
& \left(\widetilde{\nabla}_{\mathscr{P}^{*}} \eta_{*}\right)\left(\mathscr{Q}^{*}\right)=(1-\beta) \widehat{\Phi}\left(\mathscr{P}^{*}, \mathscr{Q}^{*}\right)-\alpha \ddot{g}\left(\phi_{*} \mathscr{P}^{*}, \phi_{*} \mathscr{Q}^{*}\right), \\
& \widetilde{\mathscr{R}}\left(\mathscr{P}^{*}, \mathscr{Q}^{*}\right) \widehat{\zeta}=\left(1-\beta+\beta^{2}\right)\left\{\eta_{*}\left(\mathscr{Q}^{*}\right) \mathscr{P}^{*}-\eta_{*}\left(\mathscr{P}^{*}\right) \mathscr{Q}^{*}\right\} \\
& +\alpha(1-\beta)\left\{\eta_{*}\left(\mathscr{P}^{*}\right) \phi_{*} \mathscr{Q}^{*}-\eta_{*}\left(\mathscr{Q}^{*}\right) \phi_{*} \mathscr{P}\right\}, \\
& \widetilde{\mathscr{R}}\left(\widehat{\zeta}, Q^{*}\right) \widehat{\zeta}=\left(1-\beta+\beta^{2}\right)\left\{\eta_{*}\left(\mathscr{Q}^{*}\right) \widehat{\zeta}+\mathbb{Q}^{* *}\right\}+\alpha(\beta-1) \phi_{*} \mathbb{Q}^{*}, \\
& \tilde{\mathcal{S}}\left(\mathscr{P}^{*}, \mathscr{Q}^{*}\right)=\mathcal{S}\left(\mathscr{P}^{*}, \mathscr{Q}^{*}\right)+\{-\alpha \beta+(\widehat{n}-2)(\alpha \beta-\alpha) \\
& \left.+\left(\beta^{2}-2 \beta\right) \widehat{\Phi}\right\} \widehat{\Phi}\left(\mathscr{P}^{*}, \widehat{Q}^{*}\right) \\
& +\left\{-2 \alpha^{2}+\beta-\beta^{2}+\widehat{n} \alpha^{2}+(\alpha \beta-\alpha) \widehat{\Phi}\right\} \ddot{g}\left(\mathscr{P}^{*}, Q^{*}\right) \\
& +\left\{-2 \alpha^{2}+\widehat{n}\left(\alpha^{2}+\beta-\beta^{2}\right)\right\} \eta_{*}\left(\mathscr{P}^{*}\right) \eta_{*}\left(\mathscr{Q}^{*}\right),
\end{aligned}
$$

for any $\mathscr{P}^{*}, Q^{*} \in(T \Theta)$. 


\section{CR-Submanifolds of an $\mathscr{L} \mathscr{P}$-Sasakian Manifold with GSMC- $(\alpha, \beta)$}

Here, we have recall the well-known definition in the following manner.

Definition 3 (see [23]). A Riemannian manifold $\left(\Theta^{\widehat{n}}, \ddot{g}\right)$ of an $\mathscr{L} \mathscr{P}$-Sasakian manifold $\Theta^{\widehat{n}}\left(\phi_{*}, \widehat{\zeta}, \eta, \ddot{g}\right)$ is called a $\mathscr{C} \mathscr{R}$-submanifold $(\Theta, \ddot{g})$ if $\widehat{\zeta}$ is tangent to $\Theta$ and there exists on $\Theta$ a differentiable distribution $\mathscr{D}^{*}: x \longrightarrow \mathscr{D}_{x}^{*} \subset T_{x}^{*}(\Theta)$ such that

(i) $\mathscr{D}^{*}$ is invariant under $\phi_{*}$, i.e., $\phi_{*} \mathscr{D}^{*} \subset \mathscr{D}^{*}$

(ii) The orthogonal complement distribution $\mathscr{D}^{*} \perp: x \longrightarrow \mathscr{D}_{x}^{* \perp} \subset T_{x}^{*} \Theta$ of the distribution $\mathscr{D}^{*}$ on $\Theta$ is totally real, i.e., $\phi_{*} \mathscr{D}^{* \perp} \subset T^{* \perp} \Theta$

Definition 4 (see [23]). If the distribution $\mathscr{D}^{*}$ (resp., $\mathscr{D}^{* \perp}$ ) is horizontal (resp., vertical), then the pair $\left(\mathscr{D}^{*}, \mathscr{D}^{* \perp}\right)$ is called $\widehat{\zeta}$-horizontal (resp., $\widehat{\zeta}$-vertical) if $\widehat{\zeta} \in \Gamma\left(\mathscr{D}^{*}\right)$ (resp., $\left.\zeta \in \Gamma\left(\mathscr{D}^{* \perp}\right)\right)$. The $\mathscr{C} \mathscr{R}$-submanifold is also called $\hat{\zeta}$-horizontal (resp., $\widehat{\zeta}$-vertical) if $\widehat{\zeta} \in \Gamma\left(\mathscr{D}^{*}\right)\left(\right.$ resp., $\left.\widehat{\zeta} \in \Gamma\left(\mathscr{D}^{* \perp}\right)\right)$.

The orthogonal complement $\phi_{*} \mathscr{D}^{* \perp} \in T^{* \perp} \Theta$ is given by

$$
\begin{aligned}
T^{*} \Theta & =\mathscr{D}^{*} \oplus \mathscr{D}^{* \perp}, \\
T^{* \perp} \Theta & =\phi_{*} \mathscr{D}^{* \perp} \oplus \varrho,
\end{aligned}
$$

where $\phi_{*} \varrho=\varrho$.

Let $\Theta$ be a $\mathscr{C} \mathscr{R}$-submanifold of an $\mathscr{L} \mathscr{P}$-Sasakian manifold with a GSMC- $(\alpha, \beta)$. For any $\mathscr{P}^{*} \in \Gamma\left(T^{*} \Theta\right)$ and $\mathcal{N}^{*} \in \Gamma\left(T^{* \perp} \Theta\right)$, we can write

$$
\begin{gathered}
\mathscr{P}^{*}=U \mathscr{P}^{*}+V \mathscr{P}^{*}, \quad U \mathscr{P}^{*} \in \Gamma\left(\mathscr{D}^{*}\right), V \mathscr{P}^{*} \in \Gamma\left(\mathscr{D}^{* \perp}\right), \\
\phi_{*} \mathcal{N}^{*}=B \mathscr{N}^{*}+C \mathcal{N}^{*}, \quad B \mathcal{N}^{*} \in \Gamma\left(\mathscr{D}^{* \perp}\right), C \mathcal{N}^{*} \in \Gamma(\varrho) .
\end{gathered}
$$

The Gauss and Weingarten formulas with respect to $\tilde{\nabla}$ are, respectively, given by

$$
\begin{aligned}
\tilde{\nabla}_{\mathscr{P}^{*}} Q^{*} & =\tilde{\nabla}_{\mathscr{P}^{*}} Q^{*}+\widetilde{h}\left(\mathscr{P}^{*}, Q^{*}\right), \\
\tilde{\nabla}_{\mathscr{P}^{*}} \mathcal{N}^{*} & =-\widetilde{A}_{\mathcal{N}^{*}} \mathscr{P}^{*}+\tilde{\nabla}_{\mathscr{P}^{*}}^{\perp} \mathcal{N}^{*},
\end{aligned}
$$

for any $\mathscr{P}^{*}, \mathscr{Q}^{*} \in \Gamma\left(T^{*} \Theta\right)$, where $\tilde{\nabla}_{\mathscr{P}^{*}} \mathscr{Q}^{*}, \quad \widetilde{A}_{\mathcal{N}^{*}} \mathscr{P}^{*} \in$ $\Gamma\left(T^{*} \Theta\right)$. Here, $\widetilde{\nabla}, \tilde{h}$, and $\widetilde{A}_{\mathcal{N}^{*}}$ are called the induced connection on $\Theta$, the second fundamental form, and the Weingarten mapping with respect to $\widetilde{\nabla}$, respectively. In view of (10), (12), and (17), we obtain

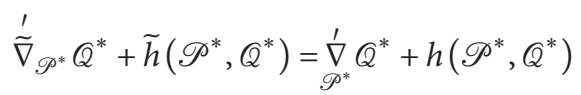

$$
\begin{aligned}
& +\alpha\left\{\eta_{*}\left(\mathscr{Q}^{*}\right) \mathscr{P}^{*}-\ddot{g}\left(\mathscr{P}^{*}, Q^{*}\right) \widehat{\zeta}\right\} \\
& +\beta\left\{\eta_{*}\left(Q^{*}\right) \phi_{*} \mathscr{P}^{*}-\ddot{g}\left(\phi_{*} \mathscr{P}^{*}, Q^{*}\right) \widehat{\zeta}\right\} .
\end{aligned}
$$

Using (15) and (16) in (19), we obtain

$$
\begin{aligned}
& \stackrel{\prime}{U \widetilde{\nabla}_{\mathscr{P}^{*}}}{\widehat{Q}^{*}}^{*} \dot{U}_{\mathscr{P}^{*}}^{\prime} \widehat{Q}^{*}+\alpha \eta_{*}\left(\widehat{Q}^{*}\right) U \mathscr{P}^{*}-\alpha \ddot{g}\left(\mathscr{P}^{*}, \widehat{Q}^{*}\right) U \widehat{\zeta} \\
& +\beta \eta_{*}\left(\mathscr{Q}^{*}\right) \phi_{*} U \mathscr{P}^{*}-\beta \ddot{g}\left(\phi_{*} \mathscr{P}^{*}, Q^{*}\right) U \widehat{\zeta}, \\
& \widetilde{h}\left(\mathscr{P}^{*}, Q^{*}\right)=h\left(\mathscr{P}^{*}, Q^{*}\right)+\beta \eta_{*}\left(\mathcal{Q}^{*}\right) \phi_{*}{Q^{*}}^{*},
\end{aligned}
$$

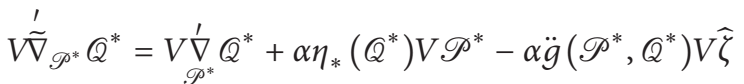

$$
\begin{aligned}
& -\beta \ddot{g}\left(\phi_{*} \mathscr{P}^{*}, \widehat{Q}^{*}\right) V \widehat{\zeta}
\end{aligned}
$$

for any $\mathscr{P}^{*}, Q^{*} \in(T \Theta)$.

\section{Quasi-Yamabe Solitons (QYS) with Torse-Forming Vector Field}

We classify QYS with torse-forming vector fields on an $\mathscr{L} \mathscr{P}$-Sasakian manifold admitting a $\operatorname{GSMC}-(\alpha, \beta)$ in this section. As a result, we can prove the theorem below.

Theorem 1. An $\mathscr{L} \mathscr{P}$-Sasakian manifold $\Theta^{\widehat{n}}\left(\phi_{*}, \widehat{\zeta}, \eta_{*}, \ddot{g}\right)$, $n>1$, with respect to GSMC- $(\alpha, \beta)$ admitting QYS. If $\mathscr{K}$ is a torse-forming vector field, then the data $(\ddot{g}, \mathscr{K}, \widehat{\sigma}, \mu)$ is growing, steadying, and contracting in accordance with $\psi-\widehat{\delta}-(1 / \widehat{n})\left\{\pi(\mathscr{K})+\alpha(\widehat{n}-1) \eta_{*}(\mathscr{K})-\mu \tau\right\} \stackrel{\leq}{=}$, unless $\psi-$ $\delta-(1 / \widehat{n})\left\{\pi(\mathscr{K})+\alpha(\widehat{n}-1) \eta_{*}(\mathscr{K})-\mu \tau\right\}$ is constant.

Proof. Let the data $(\ddot{g}, \mathscr{K}, \widehat{\sigma}, \mu)$ be a QYS on $\Theta^{\widehat{n}}\left(\phi_{*}, \widehat{\zeta}, \eta_{*}, \ddot{g}\right)$ in terms of GSMC- $(\alpha, \beta)$. From (3), we have

$$
\begin{aligned}
\frac{1}{2}\left(\widetilde{\mathfrak{Q}}_{\mathscr{K}} \ddot{\mathfrak{g}}\right)\left(\mathscr{P}^{*}, Q^{*}\right)= & (\widetilde{\widetilde{\delta}}-\widehat{\sigma}) \ddot{g}\left(\mathscr{P}^{*}, Q^{*}\right) \\
& +\mu \ddot{g}\left(\mathscr{K}^{\diamond}, \mathscr{P}^{*}\right) \ddot{g}\left(\mathscr{K}^{\diamond}, Q^{*}\right) .
\end{aligned}
$$

From (3) and (12), we obtain

$$
\begin{aligned}
\left(\widetilde{\mathfrak{I}}_{\mathscr{K}} \ddot{\mathfrak{g}}\right)\left(\mathscr{P}^{*}, \mathscr{Q}^{*}\right)= & \ddot{g}\left(\widetilde{\nabla}_{\mathscr{P}^{*}} \mathscr{K}, \mathscr{Q}^{*}\right)+\ddot{g}\left(\mathscr{P}^{*}, \widetilde{\nabla}_{\mathscr{Q}^{*}} \mathscr{K}\right) \\
= & 2 \psi \ddot{g}\left(\mathscr{P}^{*}, Y\right)+\pi\left(\mathscr{P}^{*}\right) \ddot{g}\left(\mathscr{K}, \mathscr{Q}^{*}\right)+\pi\left(\mathscr{Q}^{*}\right) \ddot{g}\left(\mathscr{K}, \mathscr{P}^{*}\right) \\
& +\alpha\left\{2 \eta_{*}(\mathscr{K}) \ddot{g}\left(\mathscr{P}^{*}, \mathscr{Q}^{*}\right)-\ddot{g}\left(\mathscr{P}^{*}, \mathscr{K}\right) \eta_{*}\left(\mathscr{Q}^{*}\right)-\ddot{g}\left(\mathscr{Q}^{*}, \mathscr{K}\right) \eta_{*}\left(\mathscr{P}^{*}\right)\right\} \\
& +\beta\left\{2 \eta_{*}(\mathscr{K}) \ddot{g}\left(\phi_{*} \mathscr{P}^{*}, \mathscr{Q}^{*}\right)-\ddot{g}\left(\phi \mathscr{P}^{*}, \mathscr{K}\right) \eta_{*}\left(\mathscr{Q}^{*}\right)-\ddot{g}\left(\phi_{*} \mathscr{Q}^{*}, \mathscr{K}\right) \eta_{*}\left(\mathscr{P}^{*}\right)\right\},
\end{aligned}
$$

for all $\mathscr{P}^{*}, Q^{*} \in \chi(\Theta)$. 
With the help of (23) and (24), we obtain

$$
\begin{aligned}
(\psi-\widetilde{\widehat{\delta}}-\widehat{\sigma}) \ddot{g}\left(\mathscr{P}^{*}, Q^{*}\right)= & \frac{1}{2}\left\{\pi\left(\mathscr{P}^{*}\right) \ddot{g}\left(\mathscr{K}, Q^{*}\right)+\pi\left(Q^{*}\right) \ddot{g}\left(\mathscr{K}, \mathscr{P}^{*}\right)\right\} \\
& +\alpha \eta_{*}(\mathscr{K}) \ddot{g}\left(\mathscr{P}^{*}, Q^{*}\right)+\beta \eta_{*}(\mathscr{K}) \ddot{g}\left(\phi_{*} \mathscr{P}^{*}, Q^{*}\right) \\
& -\frac{\alpha}{2}\left\{\ddot{g}\left(\mathscr{P}^{*}, \mathscr{K}\right) \eta_{*}\left(\mathscr{Q}^{*}\right)+\ddot{g}\left(\mathscr{Q}^{*}, \mathscr{K}\right) \eta_{*}\left(\mathscr{P}^{*}\right)\right\} \\
& -\frac{\beta}{2}\left\{\ddot{g}\left(\phi_{*} \mathscr{P}^{*}, \mathscr{K}\right) \eta_{*}\left(\mathscr{Q}^{*}\right)+\ddot{g}\left(\phi_{*} \mathscr{Q}^{*}, \mathscr{K}\right) \eta_{*}\left(\mathscr{P}^{*}\right)\right\} \\
& -\mu \ddot{g}\left(\mathscr{K}^{\diamond}, \mathscr{P}^{*}\right) \ddot{g}\left(\mathscr{K}^{\diamond}, Q^{*}\right) .
\end{aligned}
$$

On contracting (25), we find

$$
\widehat{\sigma}=\psi-\widetilde{\widehat{\delta}}-\frac{1}{\widehat{n}}\left\{\pi(\mathscr{K})+\alpha(\widehat{n}-1) \eta_{*}(\mathscr{K})-\mu \tau\right\},
$$

where $\tau=g\left(\mathscr{K}^{\diamond}, \mathscr{K}^{\diamond}\right)=\left|\mathscr{K}^{\diamond}\right|^{2}$.

As a result, Theorem 1 is proven.

In this sequel, the corollaries are as follows.

Corollary 1. If (2) defines a QYS on an $\mathscr{L} \mathscr{P}$-Sasakian manifold $\Theta^{\widehat{n}}\left(\phi_{*}, \widehat{\zeta}, \eta_{*}, \ddot{g}\right), \widehat{n}>1$, admitting a GSMC- $(\alpha, \beta)$, then there are the existing relationships representing in Table 1.

In Table $\quad 1, \quad \Omega_{1}=\psi-\widetilde{\widehat{\delta}}-(1 / \widehat{n})\{\pi(\mathscr{K})+\quad \alpha(\widehat{n}-1)$ $\left.\eta_{*}(\mathscr{K})-\mu \tau\right\}, \Omega_{2}=\psi-\widetilde{\widehat{\delta}}-(1 / \widehat{n})\left\{\alpha(\widehat{n}-1) \eta_{*}(\mathscr{K})-\mu \tau\right\}, \Omega_{3}=$ $1-\widetilde{\widehat{\delta}}-(1 / \widehat{n})\left\{\alpha(\widehat{n}-1) \eta_{*}(\mathscr{K})-\mu \tau\right\}, \quad \Omega_{4}=\widetilde{\widehat{\delta}}-(1 / \widehat{n})\{\pi(\mathscr{K})+$ $\left.\alpha(\widehat{n}-1) \eta_{*}(\mathscr{K})-\mu \tau\right\}, \quad \Omega_{5}=\widetilde{\widehat{\delta}}-(1 / n)\left\{\alpha(\widehat{n}-1) \eta_{*}(\mathscr{K})-\mu \tau\right\}$, and $\Omega_{6}=\psi-\widetilde{\widehat{\delta}}-(1 / \widehat{n})\left\{\alpha(\widehat{n}-1) \eta_{*}(\mathscr{K})-\mu \tau\right\}$.

Corollary 2. Let data $(\ddot{g}, \mathscr{K}, \widehat{\sigma}, \mu)$ be a QYS on an

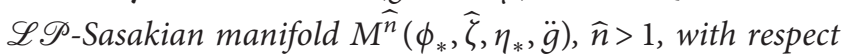
to a GSMC- $(\alpha, \beta)=(1,0)$. If $\mathscr{K}$ is torse-forming vector field, then $(\ddot{g}, \mathscr{K}, \widehat{\sigma}, \mu)$ is growing, steadying, and contracting according to $\psi-\widetilde{\widehat{\delta}}-(1 / \widehat{n})\left\{\pi(\mathscr{K})+(\widehat{n}-1) \eta_{*}(\mathscr{K})-\mu \tau\right\} \leqq 0$, unless $\quad \psi-\widetilde{\widehat{\delta}}-(1 / \widehat{n})\left\{\pi(\mathscr{K})+(\widehat{n}-1) \eta_{*}(\mathscr{K})-\mu \tau\right\} \quad$ is constant.

Corollary 3. Let $\Theta^{\hat{n}}\left(\phi_{*}, \widehat{\zeta}, \eta_{*}, \ddot{g}\right), \widehat{n}>1$, be an $\mathscr{L} \mathscr{P}$-Sasakian manifold endowed with a GSMC- $(\alpha, \beta)=(0,1)$. If a data $(\ddot{g}, \mathscr{K}, \widehat{\sigma}, \mu)$ be a QYS on $\Theta^{\widehat{n}}$ and $\mathscr{K}$ is a torse-forming vector field, then $(\ddot{g}, \mathscr{K}, \widehat{\sigma}, \mu)$ is growing, steadying, and contracting according to $\psi-\widetilde{\widetilde{\delta}}-(1 / \widehat{n})\{\pi(\mathscr{K})-\mu \tau\} \stackrel{>}{=}$, unless $\psi-\widetilde{\widehat{\delta}}-$ $(1 / \widehat{n})\{\pi(\mathscr{K})-\mu \tau\}$ is constant.

Corollary 4. If (2) defines a QYS on an $\mathscr{L} \mathscr{P}$-Sasakian manifold $\Theta^{\hat{n}}\left(\phi_{*}, \widehat{\zeta}, \eta_{*}, \ddot{g}\right), \widehat{n}>1$, with respect to a GSMC$(\alpha, \beta)=(0,1)$, then we obtain the relationship in Table 2.
Corollary 5. Let a data $(\ddot{g}, \mathscr{K}, \widehat{\sigma}, \mu)$ be a QYS on an $\mathscr{L} \mathscr{P}$-Sasakian manifold $\Theta^{\widehat{n}}\left(\phi_{*}, \widehat{\zeta}, \eta_{*}, \ddot{g}\right), \widehat{n}>1$, with respect to a GSMC- $(\alpha, \beta)=(1,0)$. Then, the following relationships are maintained in Table 3.

In Table 3, $\Omega_{7}=\psi-\widetilde{\widehat{\delta}}-(1 / \widehat{n})\left\{\pi(\mathscr{K})+(\widehat{n}-1) \eta_{*}(\mathscr{K})-\mu \tau\right\}$, $\Omega_{8}=\psi-\widetilde{\widehat{\delta}}-(1 / \widehat{n}) \quad\left\{(\widehat{n}-1) \eta_{*}(\mathscr{K})-\mu \tau\right\}, \quad \Omega_{9}=1-\widetilde{\widehat{\delta}}-(1 / \widehat{n})$ $\left\{(\widehat{n}-1) \eta_{*}(\mathscr{K})-\mu \tau\right\}, \quad \Omega_{10}=\widetilde{\widehat{\delta}}-(1 / \widehat{n})\left\{\pi(\mathscr{K})+(\widehat{n}-1) \eta_{*}(\mathscr{K})-\right.$ $\mu \tau\}, \quad \Omega_{11}=\widetilde{\widehat{\delta}}-(1 / \widehat{n})\left\{(\widehat{n}-1) \eta_{*}(\mathscr{K})-\mu \tau\right\}, \quad$ and $\quad \Omega_{12}=\psi-\widetilde{\widehat{\delta}}-$ $(1 / \widehat{n})\left\{(\widehat{n}-1) \eta_{*}(\mathscr{K})-\mu \tau\right\}$.

\section{Quasi-Yamabe Solitons (QYS) with Potential Vector Field is Torse- Forming on $\mathscr{C} \mathscr{R}$-Submanifold}

We investigate QYS in relating to a torse-forming vector field on $\mathscr{C} \mathscr{R}$-submanifolds of an $\mathscr{L} \mathscr{P}$-Sasakian manifold with regard to the induced connection $\widetilde{\nabla}$ of type $(\alpha, \beta)$ in this section. The following is our theorem.

Theorem 2. Let a $\mathscr{C} \mathscr{R}$-submanifold $\Theta$ of an $\mathscr{L} \mathscr{P}$-Sasakian manifold be $\Theta^{\hat{n}}\left(\phi_{*}, \widehat{\zeta}, \eta_{*}, \ddot{g}\right), \widehat{n}>1$, admitting a GSMC $\stackrel{1}{\widetilde{\nabla}}$ is $\widehat{\zeta}$-horizontal (resp. $\widehat{\zeta}$-vertical) and $\mathscr{D}^{*}$ is parallel with respect to $\stackrel{\prime}{\nabla}$. If data $(\ddot{g}, \mathscr{K}, \widehat{\sigma}, \mu)$ is a QYS on $\stackrel{\Theta}{\Theta}$ and $\mathscr{K}$ is a torseforming vector field, then $(\ddot{g}, \mathscr{K}, \widehat{\sigma}, \mu)$ is growing, steadying, or contracting according to $\psi-\widetilde{\widehat{\delta}}-(1 / \widehat{n})\{\pi(\mathscr{K})+$ $\left.\alpha(\widehat{n}-1) \eta_{*}(\mathscr{K})-\mu \tau\right\} \stackrel{\leq}{>} 0, \quad$ unless $\quad \psi-\widetilde{\widehat{\delta}}-(1 / \widehat{n})\{\pi(\mathscr{K})+$ $\left.\alpha(\widehat{n}-1) \eta_{*}(\mathscr{K})-\mu \tau\right\}$ is constant.

Proof. If $\Theta$ is $\widehat{\zeta}$-horizontal for all $\mathscr{P}^{*}, Q^{*} \in \Gamma\left(\mathscr{D}^{*}\right)$ and $\mathscr{D}^{*}$ is parallel in relation to $\tilde{\nabla}$, then there is, from (20),

$$
\begin{aligned}
\hat{\nabla}_{\mathscr{P}^{*}} Q^{*}= & { }_{\mathscr{P}^{*}}^{\prime} Q^{*}+\alpha\left\{\eta\left(Q^{*}\right) \mathscr{P}^{*}-g\left(\mathscr{P}^{*}, Q^{*}\right) \widehat{\zeta}\right\} \\
& +\beta\left\{\eta_{*}\left(\mathcal{Q}^{*}\right) \phi_{*} \mathscr{P}^{*}-g\left(\phi_{*} \mathscr{P}^{*}, Q^{*}\right) \widehat{\zeta}\right\} .
\end{aligned}
$$

With the help of Lemma 1 , the induced connection $\nabla$ is also a GSMC- $(\alpha, \beta)$. This leads to the statement of Theorem 2 . 
TABLE 1: Relationship between $\mathscr{K}$, existence condition, and nature of solitons.

\begin{tabular}{|c|c|c|}
\hline $\mathscr{K}$ & Existence condition & Nature of solitons \\
\hline Torse-forming & $\Omega_{1}=$ constant & $\Omega_{1} \leqq 0$ \\
\hline Concircular & $\Omega_{2}=$ constant & $\Omega_{2} \leqq 0$ \\
\hline Concurrent & $\Omega_{3}=$ constant & $\Omega_{3} \leqq 0$ \\
\hline Recurrent & $\Omega_{4}=$ constant & $\Omega_{4} \stackrel{>}{=} 0$ \\
\hline Parallel & $\Omega_{5}=$ constant & $\Omega_{5} \leqq 0$ \\
\hline Torqued & $\Omega_{6}=$ constant & $\Omega_{6} \doteq 0$ \\
\hline
\end{tabular}

TABLE 2: Relationship between $\mathscr{K}$, existence condition, and nature of solitons.

\begin{tabular}{|c|c|c|}
\hline $\mathscr{K}$ & Existence condition & Nature of solitons \\
\hline Torse-forming & $\psi-\widetilde{\widehat{\delta}}-(1 / \widehat{n})\{\pi(\mathscr{K})-\mu \tau\}=$ constant & $\psi-\widetilde{\widetilde{\delta}}-(1 / \widehat{n})\{\pi(\mathscr{K})-\mu \tau\} \leq$ \\
\hline Concircular & $\psi-\widetilde{\widehat{\delta}}-\mu \tau=$ constant & $\psi-\widetilde{\widehat{\delta}}-\mu \tau \stackrel{\leq}{>} 0$ \\
\hline Concurrent & $1-\widetilde{\widehat{\delta}}-\mu \tau=$ constant & 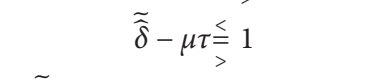 \\
\hline Recurrent & $\widetilde{\widehat{\delta}}-(1 / \widehat{n})\{\pi(\mathscr{K})-\mu \tau\}=$ constant & $\widetilde{\widetilde{\delta}}-(1 / \widehat{n})\{\pi(\mathscr{K})-\mu \tau\} \stackrel{>}{=} 0$ \\
\hline Parallel & $\widetilde{\widehat{\delta}}-\mu \tau=$ constant & $\widetilde{\widehat{\delta}}-\mu \tau \stackrel{>}{>} 0$ \\
\hline Torqued & $\psi-\widetilde{\widetilde{\delta}}-\mu \tau=$ constant & $\psi-\widetilde{\widetilde{\delta}}-\mu \tau \stackrel{\leq}{>} 0$ \\
\hline
\end{tabular}

TABLE 3: Relationship between $\mathscr{K}$, existence condition, and nature of solitons.

\begin{tabular}{|c|c|c|}
\hline $\mathscr{K}$ & Existence condition & Nature of solitons \\
\hline Torse-forming & $\Omega_{7}=$ constant & $\Omega_{7} \leqq 0$ \\
\hline Concircular & $\Omega_{8}=$ constant & $\Omega_{8} \leqq 0$ \\
\hline Concurrent & $\Omega_{9}=$ constant & $\Omega_{9} \leqq 0$ \\
\hline Recurrent & $\Omega_{10}=$ constant & $\Omega_{10} \stackrel{>}{>} 0$ \\
\hline Parallel & $\Omega_{11}=$ constant & $\Omega_{11} \stackrel{>}{>} 0$ \\
\hline Torqued & $\Omega_{12}=$ constant & $\Omega_{12} \leqq 0$ \\
\hline
\end{tabular}

In this sequel, we write the following corollaries.

Corollary 6. Let a $\mathscr{C} \mathscr{R}$-submanifold $\Theta$ of an $\mathscr{L} \mathscr{P}$-Sasakian manifold be $\Theta^{\widehat{n}}\left(\phi_{*}, \widehat{\zeta}, \eta_{*}, \ddot{g}\right), \widehat{n}>1$, admitting a GSMC $\widetilde{\nabla}$ is $\widehat{\zeta}$-horizontal (resp. $\widehat{\zeta}$-vertical) and $\mathscr{D}^{*}$ is parallel in terms of $\stackrel{\prime}{\nabla}$. If (2) defines a QYS on ${ }^{\Theta}$ and $\mathscr{K}$ is a torse-forming vector field, then the results hold in Table 4.

Corollary 7. Let a $\mathscr{C} \mathscr{R}$-submanifold $\Theta$ of an $\mathscr{L} \mathscr{P}$-Sasakian manifold be $\Theta^{\widehat{n}}\left(\phi_{*}, \widehat{\zeta}, \eta_{*}, \ddot{g}\right), \widehat{n}>1$, admitting a GSMC $\dot{\nabla}$ is $\widehat{\zeta}$-horizontal (resp. $\widehat{\zeta}$-vertical) and $\mathscr{D}^{*}$ is parallel in term of $\widetilde{\nabla}$ of type $(\alpha, \beta)=(0,1)$. If data $(\ddot{g}, \mathscr{K}, \widehat{\sigma}, \mu)$ is a QYS on $\Theta$ and $\mathscr{K}$ is a torse-forming vector field, then, in Table 5, relationships must be true.

Corollary 8. Let a $\mathscr{C} \mathscr{R}$-submanifold $\Theta$ of an $\mathscr{L} \mathscr{P}$-Sasakian manifold be $\Theta^{\widehat{n}}\left(\phi_{*}, \widehat{\zeta}, \eta_{*}, \ddot{g}\right), \widehat{n}>1$, admitting a GSMC $\dot{\nabla}$ of type $(\alpha, \beta)=(1,0)$ is $\widehat{\zeta}$-horizontal (resp. $\widehat{\zeta}$-vertical) and $\mathscr{D}^{*}$ is parallel with respect to $\tilde{\nabla}$. If $(\ddot{g}, \mathscr{K}, \widehat{\sigma}, \mu)$ is a QYS on $\Theta$ and $\mathscr{K}$ is a torse-forming vector field, then $(\ddot{g}, \mathscr{K}, \widehat{\sigma}, \mu)$ is growing, steadying, or contracting according to $\psi-\widetilde{\widehat{\delta}}-(1 / \widehat{n})\{\pi(\mathscr{K})+$ $\left.(\widehat{n}-1) \eta_{*}(\mathscr{K})-\mu \tau\right\} \stackrel{\leq}{=}, \quad$ unless $\quad \psi-\widetilde{\widehat{\delta}}-(1 / \widehat{n})\{\pi(K)+$ $\left.(\widehat{n}-1) \eta_{*}(\mathscr{K})-\mu \tau\right\}$ is constant. 
TABLE 4: Relationship between $\mathscr{K}$, existence condition, and nature of solitons.

\begin{tabular}{lcc}
\hline $\mathscr{K}$ & Existence condition & Nature of solitons \\
\hline Torse-forming & $\Omega_{1}=$ constant & $\Omega_{1} \doteq 0$ \\
Concircular & $\Omega_{2}=$ constant & $\Omega_{2} \doteq 0$ \\
Concurrent & $\Omega_{3}=$ constant & $\Omega_{3} \doteq 0$ \\
Recurrent & $\Omega_{4}=$ constant & $\Omega_{4} \doteq 0$ \\
Parallel & $\Omega_{5}=$ constant & $\Omega_{5} \doteq 0$ \\
Torqued & $\Omega_{6}=$ constant & $\Omega_{6} \leqq 0$ \\
\hline
\end{tabular}

TABLE 5: Relationship between $\mathscr{K}$, existence condition, and nature of solitons.

\begin{tabular}{|c|c|c|}
\hline $\mathscr{K}$ & Existence condition & Nature of solitons \\
\hline Torse-forming & $\Omega_{7}=$ constant & $\Omega_{7} \leftrightharpoons 0$ \\
\hline Concircular & $\Omega_{8}=$ constant & $\Omega_{8} \stackrel{\leq}{\lessgtr} 0$ \\
\hline Concurrent & $\Omega_{9}=$ constant & $\Omega_{9} \stackrel{\leq}{>} 0$ \\
\hline Recurrent & $\Omega_{10}=$ constant & $\Omega_{10} \stackrel{\leq}{>} 0$ \\
\hline Parallel & $\Omega_{11}=$ constant & $\Omega_{11} \stackrel{>}{>} 0$ \\
\hline Torqued & $\Omega_{12}=$ constant & $\Omega_{12} \stackrel{\leq}{>} 0$ \\
\hline
\end{tabular}

Corollary 9. Let a $\mathscr{C} \mathscr{R}$-submanifold $\Theta^{\Theta}$ of an $\mathscr{L} \mathscr{P}$-Sasakian manifold be $\Theta^{\hat{n}}\left(\phi_{*}, \widehat{\zeta}, \eta_{*}, \ddot{g}\right), \widehat{n}>1$, in relation to a GSMC$(\alpha, \beta)=(0,1)$ which is $\widehat{\zeta}$-horizontal (resp. $\widehat{\zeta}$-vertical) and $\mathscr{D}^{*}$ which is parallel in view og $\widetilde{\nabla}$. If $(\ddot{g}, \mathscr{K}, \widehat{\sigma}, \mu)$ is a QYS on ${ }_{\Theta} \Theta$ and $\mathscr{K}$ is a torse-forming vector field, then $(\ddot{g}, \mathscr{K}, \widehat{\sigma}, \mu)$ is growing, steadying, or shrinking according as $\psi-\widetilde{\widehat{\delta}}-(1 / \widehat{n})\{\pi(\mathscr{K})-\mu \tau\} \stackrel{\leq}{>} 0$, unless $\psi-\widetilde{\widehat{\delta}}-(1 / \widehat{n})\{\pi(\mathscr{K})-$ $\mu \tau\}$ is constant.

Corollary 10. If (2) defines a QYS on an $\mathscr{L} \mathscr{P}$-Sasakian manifold $\Theta^{n}\left(\phi_{*}, \widehat{\zeta}, \eta_{*}, \ddot{g}\right), \quad \widehat{n}>1$, concerning a GSMC$(\alpha, \beta)=(0,1)$, then, in Table 6 , relationships are true.

\section{Almost Quasi-Yamabe Solitons (AQYS) whose Potential Vector Field is Torse- Forming on $\mathscr{C} \mathscr{R}$-Submanifold}

In this section, we classify AQYS whose potential field is torse-forming on $\mathscr{C} \mathscr{R}$-submanifold of an $\mathscr{L} \mathscr{P}$-Sasakian manifold with respect to a GSMC- $(\alpha, \beta)$. At this stage, we denote $\mathscr{K}^{t}$ and $\mathscr{K}^{n}$ as tangential and normal components of such vector field. To begin, we will prove the outcome.

Theorem 3. An almost quasi-Yamabe soliton $\left(\ddot{g}, \mathscr{K}^{t}, \widehat{\sigma}, \mu\right)$ on $\mathscr{C} \mathscr{R}$-submanifold of an $\mathscr{L} \mathscr{P}$-Sasakian manifold in relation to a GSMC- $(\alpha, \beta)$ satisfies

$$
\begin{aligned}
& \left(\widehat{\delta}-\widehat{\sigma}-\psi+\eta_{*}\left(\mathscr{K}^{n}\right)\right) \ddot{g}\left(\mathscr{P}^{*}, Q^{*}\right)+\mu \ddot{g}\left(\mathscr{K}^{\diamond}, \mathscr{P}^{*}\right) \ddot{g}\left(\mathscr{K}^{\diamond}, Q^{*}\right) \\
& =\ddot{g}\left(A_{\mathscr{K}^{n}}^{n} \mathscr{P}^{*}, Q^{*}\right) \\
& +\frac{1}{2}\left\{\pi\left(\mathscr{P}^{*}\right) \ddot{g}\left(\mathscr{K}, Q^{*}\right)+\pi\left(\mathscr{Q}^{*}\right) \ddot{g}\left(\mathscr{P}^{*}, \mathscr{K}\right)\right\} \\
& +\frac{\beta}{2}\left\{\ddot{g}\left(\mathscr{K}^{n}, \phi_{*} \mathscr{P}^{*}\right) \eta_{*}\left(\mathscr{Q}^{*}\right)+\ddot{g}\left(\phi_{*} \mathscr{Q}^{*}, \mathscr{K}^{n}\right) \eta_{*}\left(\mathscr{P}^{*}\right)\right\},
\end{aligned}
$$

any type of vector field $\mathscr{P}^{*}, \mathcal{Q}$ on $\Theta$.

Proof. In light of (3), (12), (17), and (18), we have

$$
\begin{aligned}
\psi \mathscr{P}^{*}+\pi\left(P^{*}\right) \mathscr{K}= & \widetilde{\nabla}_{\mathscr{P}^{*}} \mathscr{K}=\widetilde{\nabla}_{\mathscr{P}^{*}}\left(\mathscr{K}^{t}+\mathscr{K}^{n}\right)=\bar{\nabla}_{\mathscr{P}^{*}} \mathscr{K}^{t}+h\left(\mathscr{P}^{*}, \mathscr{K}^{t}\right)+\beta \eta_{*}\left(\mathscr{K}^{t}\right) \phi_{*} \mathscr{Q}^{*} \mathscr{P}^{*} \\
& -A_{\mathscr{K}^{n}} \mathscr{P}^{*}+\nabla_{\mathscr{P}^{*}}^{\perp} \mathscr{K}^{n}+\alpha \eta_{*}\left(\mathscr{K}^{n}\right) \mathscr{P}^{*}+\beta \eta_{*}\left(\mathscr{K}^{n}\right) \phi_{*} \mathscr{P}^{*}-\beta \ddot{g}\left(\phi_{*} \mathscr{P}^{*}, \mathscr{K}^{n}\right) \widehat{\zeta} .
\end{aligned}
$$

We obtain the following equation when we compare the tangential and normal components of (29):

$$
\begin{aligned}
\nabla_{\mathscr{P}^{*}} & \mathscr{K}^{t}=\psi \mathscr{P}^{*}+\pi\left(\mathscr{P}^{*}\right) K+A_{\mathscr{K}^{n}} \mathscr{P}^{*}-\alpha \eta\left(\mathscr{K}^{n}\right) \mathscr{P}^{*}-\beta \eta_{*}\left(\mathscr{K}^{n}\right) \phi_{*} \mathscr{P}^{*}+\beta \ddot{g}\left(\phi_{*} \mathscr{P}^{*}, \mathscr{K}^{n}\right) \widehat{\zeta}, \\
h\left(\mathscr{P}^{*}, \mathscr{K}^{t}\right) & =-\nabla_{\mathscr{P}^{*}}^{\perp} \mathscr{K}^{n}-\beta \eta_{*}\left(\mathscr{K}^{n}\right) \phi_{*} \mathscr{Q}^{*} \mathscr{P}^{*} .
\end{aligned}
$$

We may deduce from the concept of Lie derivative and (31) that 
TABLE 6: Relationship between $\mathscr{K}$, existence condition, and nature of solitons.

\begin{tabular}{|c|c|c|}
\hline $\mathscr{K}$ & Existence condition & Nature of solitons \\
\hline Torse-forming & $\psi-\widetilde{\widehat{\delta}}-(1 / \widehat{n})\{\pi(\mathscr{K})-\mu \tau\}=$ constant & $\psi-\widetilde{\widetilde{\delta}}-(1 / \widehat{n})\{\pi(\mathscr{K})-\mu \tau\} \stackrel{\leqq}{=} 0$ \\
\hline Concircular & $\psi-\widetilde{\widetilde{\delta}}-\mu \tau=$ constant & $\psi-\widetilde{\widetilde{\delta}}-\mu \tau \leqq 0$ \\
\hline Concurrent & $1-\tilde{\widetilde{\delta}}-\mu \tau=$ constant & $\tilde{\widetilde{\delta}}-\mu \tau \stackrel{\leqq}{>} 1$ \\
\hline Recurrent & $\widetilde{\widetilde{\delta}}-(1 / \widehat{n})\{\pi(\mathscr{K})-\mu \tau\}=$ constant & $\widetilde{\widetilde{\delta}}-(1 / \widehat{n})\{\pi(\mathscr{K})-\mu \tau\} \leqq 0$ \\
\hline Parallel & $\tilde{\widetilde{\delta}}-\mu \tau=$ constant & $\tilde{\widetilde{\delta}}-\mu \tau \stackrel{\leq}{>} 0$ \\
\hline Torqued & $\psi-\widetilde{\widetilde{\delta}}-\mu \tau=$ constant & $\psi-\widetilde{\widetilde{\delta}}-\mu \tau \triangleq 0$ \\
\hline
\end{tabular}

$$
\begin{aligned}
\mathfrak{S}_{\mathscr{K}^{t}} \ddot{\mathfrak{g}}\left(\mathscr{P}^{*}, \mathscr{Q}^{*}\right)= & 2 \psi \ddot{g}\left(\mathscr{P}^{*}, Q^{*}\right)+2 \ddot{g}\left(A_{\mathscr{K}}^{n} \mathscr{P}^{*}, \mathscr{Q}^{*}\right)-2 \eta_{*}\left(\mathscr{K}^{n}\right) \ddot{g}\left(\mathscr{P}^{*}, \mathscr{Q}^{*}\right) \\
& +\pi\left(\mathscr{P}^{*}\right) \ddot{g}\left(\mathscr{K}, \mathscr{Q}^{*}\right)+\pi\left(\mathscr{Q}^{*}\right) \ddot{g}\left(\mathscr{P}^{*}, \mathscr{K}\right)+\beta\left\{\ddot{g}\left(\mathscr{K}^{n}, \phi_{*} \mathscr{P}^{*}\right) \eta_{*}\left(\mathscr{Q}^{*}\right)\right. \\
& \left.+\ddot{g}\left(\phi \mathscr{Q}^{*}, \mathscr{K}^{n}\right) \eta_{*}\left(\mathscr{P}^{*}\right)\right\} .
\end{aligned}
$$

Using (32) in (2), we yield

$\left(\widehat{\delta}-\widehat{\sigma}-\psi+\eta_{*}\left(\mathscr{K}^{n}\right)\right) \ddot{g}\left(\mathscr{P}^{*}, \mathscr{Q}^{*}\right)+\mu \widehat{g}\left(\mathscr{K}^{\diamond}, \mathscr{P}^{*}\right) \ddot{g}\left(\mathscr{K}^{\diamond}, \mathscr{Q}^{*}\right)=\ddot{g}\left(A_{\mathscr{K}}^{n} \mathscr{P}^{*}, \mathscr{Q}^{*}\right)$

$$
\begin{aligned}
+ & \frac{1}{2}\left\{\pi\left(\mathscr{P}^{*}\right) \ddot{g}\left(\mathscr{K}, \mathscr{Q}^{*}\right)+\pi\left(\mathscr{Q}^{*}\right) \ddot{g}\left(\mathscr{P}^{*}, \mathscr{K}\right)\right\} \\
+ & \frac{\beta}{2}\left\{\ddot{g}\left(\mathscr{K}^{n}, \phi_{*} \mathscr{P}^{*}\right) \eta_{*}\left(\mathscr{Q}^{*}\right)+\ddot{g}\left(\phi_{*} \mathscr{Q}^{*}, \mathscr{K}^{n}\right) \eta_{*}\left(\mathscr{P}^{*}\right)\right\} . \\
& \left(\widehat{\delta}-\widehat{\sigma}-\psi+\eta_{*}\left(\mathscr{K}^{n}\right)\right) \hat{n}+\mu \tau=\pi(\mathscr{K}) .
\end{aligned}
$$

This completed our assertion.

As a result, the following corollaries are stated.

Corollary 11. If (2) defines AQYS on $\mathscr{C} \mathscr{R}$-submanifold of an $\mathscr{L} \mathscr{P}$-Sasakian manifold in relation to a GSMC- $(\alpha, \beta)$ which is minimal, consequently, the following relationship holds:

Corollary 12. If (2) defines AQYS on $\mathscr{C} \mathscr{R}$-submanifold of an $\mathscr{L} \mathscr{P}$-Sasakian manifold and the distribution is $\bar{\zeta}$-horizontal (resp. $\widehat{\zeta}$-vertical), $\mathscr{P}^{*}{ }_{3} \mathscr{Q}^{*} \in \Gamma\left(\mathscr{D}^{*}\right)$, where $\mathscr{D}^{*}$ is parallel with induced connection $\nabla$ of type $(\alpha, \beta)$, then we have

$$
\begin{aligned}
\left(\widehat{\delta}-\widehat{\sigma}-\psi+\eta_{*}\left(\mathscr{K}^{n}\right)\right) \ddot{g}\left(\mathscr{P}^{*}, \mathscr{Q}^{*}\right)+\mu \ddot{g}\left(\mathscr{K}^{\diamond}, \mathscr{P}^{*}\right) \ddot{g}\left(\mathscr{K}^{\diamond}, \mathscr{Q}^{*}\right)= & \ddot{g}\left(A_{\mathscr{K}}^{n} \mathscr{P}^{*}, \mathscr{Q}^{*}\right) \\
& +\frac{1}{2}\left\{\pi\left(\mathscr{P}^{*}\right) \ddot{g}\left(\mathscr{K}, \mathscr{Q}^{*}\right)+\pi\left(\mathscr{Q}^{*}\right) \ddot{g}\left(\mathscr{P}^{*}, \mathscr{K}\right)\right\} \\
& +\frac{\beta}{2}\left\{\ddot{g}\left(\mathscr{K}^{n}, \phi_{*} \mathscr{P}^{*}\right) \eta_{*}\left(\mathscr{Q}^{*}\right)+\ddot{g}\left(\phi_{*} \mathscr{Q}^{*}, \mathscr{K}^{n}\right) \eta_{*}\left(\mathscr{P}^{*}\right)\right\}, \\
\text { in all vector fields } \mathscr{P}^{*}, \mathscr{Q}^{*} \text { on } \Theta . & \left(\widehat{\delta}-\widehat{\sigma}-\psi+\eta_{*}\left(\mathscr{K}^{n}\right)\right) \widehat{n}+\mu \tau=\pi(\mathscr{K}) .
\end{aligned}
$$

Corollary 13. If data $\left(\ddot{g}, \mathscr{K}^{t}, \widehat{\sigma}, \mu\right)$ is a AQYS on $\mathscr{C} \mathscr{R}$-submanifold of an $\mathscr{L} \mathscr{P}$-Sasakian manifold and the distribution is $\bar{\zeta}$-horizontal (resp. $\widehat{\zeta}$-vertical), $\mathscr{P}^{*}, \mathscr{Q}^{*} \in \Gamma\left(\mathscr{D}^{*}\right), \mathscr{D}^{*}$ is parallel with induced connection $\nabla$ of type $(\alpha, \beta)$ is minimal, then the relation holds:

\section{Example}

A 4-dimensional differentiable manifold is taken into consideration, that is, $\Theta^{4}=\left\{(p, q, z, t) \in \mathfrak{R}^{4}\right.$ : $(p, q, z, t) \neq 0\}$, where $(p, q, z, t)$ is the standard coordinate 
in $\mathfrak{R}^{4}$. At each point along $\Theta^{4},\left(\breve{e}_{1}, \breve{e}_{2}, \breve{e}_{3}, \breve{e}_{4}\right)$ is a set of linearly independent vector fields and is described as

$$
\begin{aligned}
& \breve{e}_{1}=e^{p-t} \frac{\partial}{\partial p} \\
& \breve{e}_{2}=e^{q-t} \frac{\partial}{\partial q} \\
& \breve{e}_{3}=e^{z-t} \frac{\partial}{\partial z} \\
& \breve{e}_{4}=\frac{\partial}{\partial t} .
\end{aligned}
$$
follows:

Also, the Lie bracket's nonvanishing components are as

$$
\begin{aligned}
& {\left[\breve{e}_{1}, \breve{e}_{4}\right]=\breve{e}_{1},} \\
& {\left[\breve{e}_{2}, \breve{e}_{4}\right]=\breve{e}_{2},} \\
& {\left[\breve{e}_{3}, \breve{e}_{4}\right]=\breve{e}_{3} .}
\end{aligned}
$$

Let $\ddot{g}$ on $\Theta$ be the Lorentzian metric as

$$
\ddot{g}_{i j}=\ddot{g}\left(\breve{e}_{i}, \breve{e}_{j}\right)= \begin{cases}0, & \text { if } i \neq j, \\ -1, & \text { if } i=j=4, \\ 1, & \text { otherwise. }\end{cases}
$$

Let $\eta_{*}$ be the 1 -form corresponding to the Lorentzian metric $\ddot{g}$ :

$$
\eta_{*}\left(\mathscr{P}^{*}\right)=\ddot{g}\left(\mathscr{P}^{*}, \breve{e}_{4}\right),
$$

for any $\mathscr{P}^{*} \in \Gamma(\Theta)$. If $\phi_{*}$ is defined as the $(1,1)$-tensor field,

$$
\begin{aligned}
& \phi_{*}\left(\breve{e}_{1}\right)=\breve{e}_{1}, \\
& \phi_{*}\left(\breve{e}_{2}\right)=\breve{e}_{2}, \\
& \phi_{*}\left(\breve{e}_{3}\right)=\breve{e}_{3}, \\
& \phi_{*}\left(\breve{e}_{4}\right)=0 .
\end{aligned}
$$

We can readily prove this using the linearity characteristics of $\phi_{*}$ and $\ddot{g}$ :

$$
\begin{aligned}
\eta_{*}\left(\breve{e}_{4}\right) & =-1, \\
\phi_{*}^{2}\left(\mathscr{P}^{*}\right) & =\mathscr{P}^{*}+\eta_{*}\left(\mathscr{P}^{*}\right) \breve{e}_{4}, \\
\ddot{g}\left(\phi_{*} \mathscr{P}^{*}, \phi_{*} \mathscr{Q}^{*}\right) & =\ddot{g}\left(\mathscr{P}^{*}, \mathscr{Q}^{*}\right)+\eta_{*}\left(\mathscr{P}^{*}\right) \eta_{*}\left(\mathscr{Q}^{*}\right),
\end{aligned}
$$

for any $\mathscr{P}^{*}, \mathscr{Q}^{*} \in \Gamma(T \Theta)$. Thus, for $\breve{e}_{4}=\widehat{\zeta}$, the frame $\left(\phi_{*}, \widehat{\zeta}, \eta_{*}, \ddot{g}\right)$ leads to an $\mathscr{L} \mathscr{P}$-contact skeleton, which is known as the $\mathscr{L} \mathscr{P}$-contact manifold of dimension 4 . Now, for $\breve{e}_{4}=\widehat{\zeta}$, Koszul's formula gives the nonvanishing component:

$$
\begin{aligned}
& \nabla_{\breve{e}_{1}} \breve{e}_{1}=\breve{e}_{4}, \\
& \nabla_{\breve{e}_{1}} \breve{e}_{4}=\breve{e}_{1}, \\
& \nabla_{\breve{e}_{2}} \breve{e}_{2}=\breve{e}_{4}, \\
& \nabla_{\breve{e}_{2}} \breve{e}_{4}=\breve{e}_{2}, \\
& \nabla_{\breve{e}_{3}} \breve{e}_{3}=\breve{e}_{4}, \\
& \nabla_{\breve{e}_{3}} \breve{e}_{4}=e_{3} .
\end{aligned}
$$

Using the above equation, it can be easily verified that $\nabla_{\mathscr{P}^{*}} \widetilde{e}_{4}=\phi_{*} \mathscr{P}^{*}$ holds for each $\mathscr{P}^{*} \in \chi(\Theta)$. Thus, an $\mathscr{L} \mathscr{P}$-contact manifold is a 4 -dimensional $\mathscr{L} \mathscr{P}$-Sasakian manifold. From (12), we calculate as follows:

$$
\begin{aligned}
& \widetilde{\nabla}_{\breve{e}_{1}} \breve{e}_{1}=(1-\alpha-\beta) \breve{e}_{4}, \\
& \widetilde{\nabla}_{\breve{e}_{1}} \breve{e}_{4}=(1-\alpha-\beta) \breve{e}_{1}, \\
& \widetilde{\nabla}_{\breve{e}_{2}} \breve{e}_{2}=(1-\alpha-\beta) \breve{e}_{4}, \\
& \widetilde{\nabla}_{\breve{e}_{2}} \breve{e}_{4}=(1-\alpha-\beta) \breve{e}_{2}, \\
& \widetilde{\nabla}_{\breve{e}_{3}} \breve{e}_{3}=(1-\alpha-\beta) \breve{e}_{4}, \\
& \widetilde{\nabla}_{\breve{e}_{3}} \breve{e}_{4}=(1-\alpha-\beta) \breve{e}_{3} .
\end{aligned}
$$

It is clear from $(12)$ that $\widetilde{\nabla} \mathscr{P}^{*} \breve{e}_{4}=(1-\beta) \phi_{*} \mathscr{P}^{*}-\alpha \mathscr{P}^{*}-$ $\alpha \eta_{*}\left(\mathscr{P}^{*}\right) \breve{e}_{4}$ holds for each $\mathscr{P}^{*} \in \chi(\Theta)$. So, an $\mathscr{L} \mathscr{P}$-Sasakian manifold admitted a GSMC- $(\alpha, \beta)$.

The nonvanishing components of the curvature tensor using the aforementioned formulas are

$$
\begin{aligned}
& \widetilde{\mathscr{R}}\left(\breve{e}_{1}, \breve{e}_{2}\right) \breve{e}_{1}=-(1-\alpha-\beta)^{2} \breve{e}_{2}, \\
& \widetilde{\mathscr{R}}\left(\breve{e}_{1}, \breve{e}_{3}\right) \breve{e}_{1}=-(1-\alpha-\beta)^{2} \breve{e}_{3}, \\
& \widetilde{\mathscr{R}}\left(\breve{e}_{1}, \breve{e}_{4}\right) \breve{e}_{1}=-(1-\alpha-\beta) \breve{e}_{4}, \\
& \widetilde{\mathscr{R}}\left(\breve{e}_{1}, \breve{e}_{2}\right) \breve{e}_{2}=(1-\alpha-\beta)^{2} \breve{e}_{1}, \\
& \widetilde{\mathscr{R}}\left(\breve{e}_{2}, \breve{e}_{3}\right) \breve{e}_{2}=-(1-\alpha-\beta)^{2} \breve{e}_{3}, \\
& \widetilde{\mathscr{R}}\left(\breve{e}_{2}, \breve{e}_{4}\right) \breve{e}_{2}=-(1-\alpha-\beta) \breve{e}_{4}, \\
& \widetilde{\mathscr{R}}\left(\breve{e}_{1}, \breve{e}_{3}\right) \breve{e}_{3}=(1-\alpha-\beta)^{2} \breve{e}_{1}, \\
& \widetilde{\mathscr{R}}\left(\breve{e}_{2}, \breve{e}_{3}\right) \breve{e}_{3}=(1-\alpha-\beta)^{2} \breve{e}_{2}, \\
& \widetilde{\mathscr{R}}\left(\breve{e}_{3}, \breve{e}_{4}\right) \breve{e}_{3}=-(1-\alpha-\beta) \breve{e}_{4}, \\
& \widetilde{\mathscr{R}}\left(\breve{e}_{1}, \breve{e}_{4}\right) \breve{e}_{4}=-(1-\alpha-\beta) \breve{e}_{1}, \\
& \widetilde{\mathscr{R}}\left(\breve{e}_{2}, \breve{e}_{4}\right) \breve{e}_{4}=-(1-\alpha-\beta) \breve{e}_{2}, \\
& \widetilde{\mathscr{R}}\left(\breve{e}_{3}, \breve{e}_{4}\right) \breve{e}_{4}=-(1-\alpha-\beta) \breve{e}_{3} .
\end{aligned}
$$

The Ricci tensor of $\Theta$ is defined as $\tilde{\mathcal{S}}\left(\mathscr{P}^{*}, Q^{*}\right)=$ $\sum_{i=1}^{4} \varepsilon_{i} \ddot{g}\left(\widetilde{\mathscr{R}}\left(\breve{e}_{i}, \mathscr{P}^{*}\right) \mathscr{Q}^{*}, \breve{e}_{i}\right)$, where $\varepsilon_{i}=\ddot{g}\left(\breve{e}_{i}, \breve{e}_{i}\right)$, and is given 


$$
\tilde{\mathcal{S}}\left(\breve{e}_{i}, \breve{e}_{j}\right)=\left[\begin{array}{cccc}
3(1-\alpha-\beta)^{2} & 0 & 0 & 0 \\
0 & 3(1-\alpha-\beta)^{2} & 0 & 0 \\
0 & 0 & 3(1-\alpha-\beta)^{2} & 0 \\
0 & 0 & 0 & -3(1-\alpha-\beta)^{2}
\end{array}\right] .
$$

Also, the scalar curvature $\widetilde{\widehat{\delta}}=\sum_{i=1}^{4} \widetilde{\mathcal{S}}\left(\breve{e}_{i}, \breve{e}_{j}\right)=$ $6(1-\alpha-\beta)^{2}$.

Let any vector fields $\mathscr{P}^{*}, \mathscr{Q}^{*}$, and $\mathscr{Z}^{*} \in \chi\left(\Theta^{4}\right)$; it is possible to write

$$
\begin{aligned}
& \mathscr{P}^{*}=a_{1} \breve{e}_{1}+b_{1} \breve{e}_{2}+c_{1} \breve{e}_{3}+d_{1} \breve{e}_{4}, \\
& \mathscr{Q}^{*}=a_{2} \breve{e}_{1}+b_{2} \breve{e}_{2}+c_{2} \breve{e}_{3}+d_{2} \breve{e}_{4}, \\
& \mathscr{Z}=a_{3} \breve{e}_{1}+b_{3} \breve{e}_{2}+c_{3} \breve{e}_{3}+d_{3} \breve{e}_{4},
\end{aligned}
$$

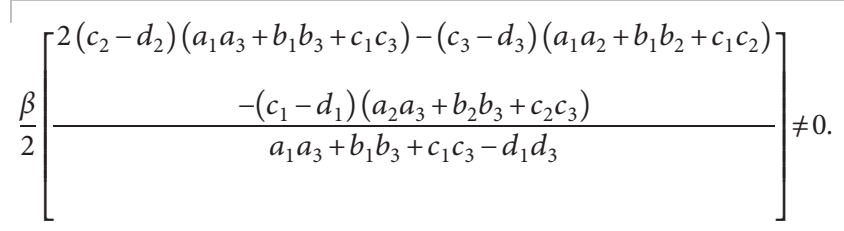

$$
\begin{aligned}
& \text { If we consider the } 1 \text {-form } \pi \text { by } \\
& \pi\left(\mathscr{P}^{*}\right)=\ddot{g}\left(\mathscr{P}^{*},(1-\alpha-\beta) \breve{e}_{4}\right) \text {, for any } \mathscr{P}^{*} \in \chi(\Theta) \text { and } \\
& \text { considering } \psi \in C^{\infty}(\Theta) \text { as }
\end{aligned}
$$

where $a_{i}, b_{i}, c_{i}, d_{i} \in \mathfrak{R}^{+}, i=1,2,3,4$, in order for

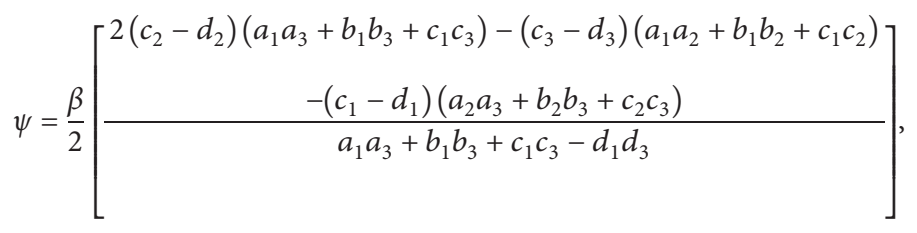

therefore, the relation,

$$
\nabla_{\mathscr{P}^{*}} \mathscr{Q}^{*}=\psi \mathscr{P}^{*}+\pi\left(\mathscr{P}^{*}\right) \mathscr{Q}^{*}
$$

holds. As per these consequences, from (24), we obtain

$$
\begin{aligned}
\left(\widetilde{\mathfrak{Q}}_{\mathscr{Q}^{*}} \ddot{\mathfrak{g}}\right)\left(\mathscr{P}^{*}, \mathscr{Z}^{*}\right)= & \ddot{g}\left(\widetilde{\nabla} \mathscr{P}^{*} \mathscr{Q}^{*}, \mathscr{Z}^{*}\right)+\ddot{g}\left(\mathscr{P}^{*}, \tilde{\nabla}_{\mathscr{Z}^{*}} \mathscr{Q}^{*}\right) \\
= & 2 \psi \ddot{g}\left(\mathscr{P}^{*}, \mathscr{Z}^{*}\right)+\pi\left(\mathscr{P}^{*}\right) \ddot{g}\left(\mathscr{Q}^{*}, \mathscr{Z}^{*}\right)+\pi\left(\mathscr{Z}^{*}\right) \ddot{g}\left(\mathscr{Q}^{*}, \mathscr{P}^{*}\right) \\
& +\alpha\left\{2 \eta_{*}\left(\mathscr{Q}^{*}\right) \ddot{g}\left(\mathscr{P}^{*}, \mathscr{Z}^{*}\right)-\ddot{g}\left(\mathscr{P}^{*}, \mathscr{Q}^{*}\right) \eta_{*}\left(\mathscr{Z}^{*}\right)-\ddot{g}\left(\mathscr{Z}^{*}, \mathscr{Q}^{*}\right) \eta_{*}\left(\mathscr{P}^{*}\right)\right\} \\
& +\beta\left\{2 \eta_{*}\left(\mathscr{Q}^{*}\right) \ddot{g}\left(\phi_{*} \mathscr{P}^{*}, \mathscr{Z}^{*}\right)-\ddot{g}\left(\phi_{*} \mathscr{P}^{*}, \mathscr{Q}^{*}\right) \eta_{*}\left(\mathscr{Z}^{*}\right)-\ddot{g}\left(\phi_{*} \mathscr{Z}^{*}, \mathscr{Q}^{*}\right) \eta_{*}\left(\mathscr{P}^{*}\right)\right\} .
\end{aligned}
$$

Also, we calculate

$$
\begin{aligned}
& \ddot{g}\left(\mathscr{P}^{*}, \mathscr{Z}^{*}\right)=a_{1} a_{3}+b_{1} b_{3}+c_{1} c_{3}-d_{1} d_{3}, \\
& \ddot{g}\left(\mathscr{Q}^{*}, \mathscr{Z}^{*}\right)=a_{2} a_{3}+b_{2} b_{3}+c_{2} c_{3}-d_{2} d_{3}, \\
& \ddot{g}\left(\mathscr{P}^{*}, \mathscr{Q}^{*}\right)=a_{1} a_{2}+b_{1} b_{2}+c_{1} c_{2}-d_{1} d_{2} .
\end{aligned}
$$

$$
\begin{aligned}
\pi\left(\mathscr{P}^{*}\right) & =-(1-\alpha-\beta) d_{1}, \\
\pi\left(\mathscr{Q}^{*}\right) & =-(1-\alpha-\beta) d_{2}, \\
\pi\left(\mathscr{Z}^{*}\right) & =-(1-\alpha-\beta) d_{3} .
\end{aligned}
$$

With the help of (52) and (53), equation (51) reduces

Also,

$$
\begin{aligned}
\frac{1}{2}\left(\mathfrak{Q}_{\mathbb{Q}^{*}} \ddot{g}\right)\left(\mathscr{P}^{*}, \mathscr{Z}^{*}\right)= & \psi\left\{a_{1} a_{3}+b_{1} b_{3}+c_{1} c_{3}-d_{1} d_{3}\right\}-\frac{1}{2}(1-\alpha-\beta)\left\{d _ { 1 } \left(a_{2} a_{3}\right.\right. \\
& \left.\left.+b_{2} b_{3}+c_{2} c_{3}-d_{2} d_{3}\right)+d_{3}\left(a_{1} a_{2}+b_{1} b_{2}+c_{1} c_{2}-d_{1} d_{2}\right)\right\}+\frac{\alpha}{2}\left\{2 ( c _ { 2 } - d _ { 2 } ) \left(a_{1} a_{3}\right.\right. \\
& \left.+b_{1} b_{3}+c_{1} c_{3}-d_{1} d_{3}\right)-\left(c_{3}-d_{3}\right)\left(a_{1} a_{2}+b_{1} b_{2}+c_{1} c_{2}-d_{1} d_{2}\right) \\
& \left.-\left(c_{1}-d_{1}\right)\left(a_{2} a_{3}+b_{2} b_{3}+c_{2} c_{3}-d_{2} d_{3}\right)\right\}+\frac{\beta}{2}\left\{2\left(c_{2}-d_{2}\right)\left(a_{1} a_{3}+b_{1} b_{3}+c_{1} c_{3}\right)\right. \\
& \left.-\left(c_{3}-d_{3}\right)\left(a_{1} a_{2}+b_{1} b_{2}+c_{1} c_{2}\right)-\left(c_{1}-d_{1}\right)\left(a_{2} a_{3}+b_{2} b_{3}+c_{2} c_{3}\right)\right\} .
\end{aligned}
$$


Also,

$$
\begin{aligned}
(\widetilde{\widetilde{\delta}}-\widehat{\sigma}) \ddot{g}\left(\mathscr{P}^{*}, \mathscr{Z}^{*}\right)= & \left.\left.\left(6(1-\alpha-\beta)^{2}\right)-\widehat{\sigma}\right)\right) \\
& \cdot\left\{a_{1} a_{3}+b_{1} b_{3}+c_{1} c_{3}-d_{1} d_{3}\right\} .
\end{aligned}
$$

We consider that $a_{1} a_{3}+b_{1} b_{3}+c_{1} c_{3}-d_{1} d_{3} \neq 0,2 d_{1}\left(a_{2}\right.$ $\left.a_{3}+b_{2} b_{3}+c_{2} c_{3}-d_{2} d_{3}\right)+2 d_{3}\left(a_{1} a_{2}+b_{1} b_{2}+c_{1} c_{2}-d_{1} d_{2}\right)+$ $d_{2}\left(a_{1} a_{3}+b_{1} b_{3}+c_{1} c_{3}-d_{1} d_{3}\right)=0$, and $-2\left(c_{3}-d_{3}\right)\left(a_{1} a_{2}+\right.$ $\left.b_{1} b_{2}+c_{1} c_{2}-d_{1} d_{2}\right)-2\left(c_{1}-d_{1}\right)\left(a_{2} a_{3}+b_{2} b_{3}+c_{2} c_{3}-d_{2} d_{3}\right)-$ $3\left(c_{2}-d_{2}\right)\left(a_{1} a_{3}+b_{1} b_{3}+c_{1} c_{3}-d_{1} d_{3}\right)=0$.

Thus, we get $\left(\ddot{g}, Q^{*}, \widehat{\sigma}\right)$ is a Yamabe soliton, that is, $(1 / 2) \mathfrak{Q}_{Q^{*}} \ddot{g}\left(\mathscr{P}^{*}, \mathscr{Z}^{*}\right)=(\widetilde{\widetilde{\delta}}-\widehat{\sigma}) \ddot{g}\left(\mathscr{P}^{*}, \mathscr{Z}^{*}\right)$ holds, unless

$$
\begin{aligned}
\widehat{\sigma} & =\psi-6(1-\alpha-\beta)^{2}-\frac{1}{4}\left\{-(1-\alpha-\beta) d_{2}+3 \alpha\left(c_{2}-d_{2}\right)\right\} \\
& =\psi-\widetilde{\widehat{\delta}}-\frac{1}{4}\left\{\pi\left(Q^{*}\right)+3 \alpha \eta_{*}\left(Q^{*}\right)\right\}=\text { constant. }
\end{aligned}
$$

As a result, the existence of the YS $\left(\ddot{g}, Q^{*}, \widehat{\sigma}\right)$ on a 4 dimensional $\mathscr{L} \mathscr{P}$-Sasakian manifold admitting a GSMC$(\alpha, \beta)$ with potential vector field $Q^{*}$ as torse-forming is justified. Then, Theorems 1 and 2 are verified.

\section{Data Availability}

No data were used to support this study.

\section{Conflicts of Interest}

There are no conflicts of interest regarding the publication of this article.

\section{References}

[1] R. S. Hamilton, "The Ricci flow on surfaces," Contemporary Mathematics, vol. 71, pp. 237-262, 1998.

[2] L. F. Di Cerbo and M. M. Disconzi, "Yamabe solitons, determinant of the laplacian and the uniformization theorem for riemann surfaces," Letters in Mathematical Physics, vol. 83, no. 1, pp. 13-18, 2008.

[3] B.-Y. Chen and S. Deshmukh, "Yamabe and quasi-Yamabe solitons on Euclidean submanifolds," Mediterranean Journal of Mathematics, vol. 15, no. 5, p. 194, 2018.

[4] G. Huang and H. Li, "On a classification of the quasi Yamabe gradient solitons," Methods and Applications of Analysis, vol. 21, no. 3, pp. 379-390, 2014.

[5] B. L. Neto and H. P. De Oliveira, "Generalized quasi Yamabe gradient solitons," Differential Geometry and its Applications, vol. 49, pp. 167-175, 2016.

[6] X. Chen, "Almost quasi-Yamabe solitons on almost cosymplectic manifolds," International Journal of Geometric Methods in Modern Physics, vol. 17, no. 5, p. 2050070, 2020.

[7] V. Pirhadi and A. Razavi, "On the almost quasi-Yamabe solitons," International Journal of Geometric Methods in Modern Physics, vol. 14, p. 1750161, 2017.

[8] K. De and U. C. De, "Almost quasi-Yamabe solitons and gradient almost quasi-yamabe solitons in paracontact geometry," Quaestiones Mathematicae, pp. 1-12, 2020.

[9] S. Ghosh, U. C. De, and A. Yildiz, "A note on almost quasi Yamabe solitons and gradient almost quasi Yamabe solitons,"
Hacettepe Journal of Mathematics and Statistics, vol. 50, no. 3 , pp. $770-777,2021$.

[10] J.-B. Jun and M. Danish Siddiqi, "Almost quasi-Yamabe soliton on (LCS) ${ }_{n}$-manifolds," Honam Mathematical Journal, vol. 42, no. 3, pp. 521-536, 2020.

[11] K. De and U. C. De, "Almost quasi-Yamabe and gradient almost quasi-Yamabe soliton on quasi-Sasakian manifolds," Acta Mathematica Universitatis Comenianae, vol. 90, no. 3, pp. 333-340, 2021.

[12] S. K. Yadav, O. Bahadir, and S. K. Chaubey, "Almost Yamabe solitons on LP-Sasakian manifolds with generalized symmetric metric connection of type $(\alpha, \beta)$," Balkan Journal of Geometry and its Applications, vol. 25, no. 2, pp. 124-139, 2020.

[13] A. M. Blagaa, "A note onWarped product almost quasiyamabe solitons," Filomat, vol. 33, no. 7, pp. 2009-2016, 2019.

[14] K. Yano, "On torse-forming direction in a Riemannian space," Proceedings of the Imperial Academy Tokyo, vol. 20, pp. 340-345, 1944.

[15] K. Yano, "On semi-symmetric metric connection," Revue Roumaine de Mathématique Pures et Appliquées, vol. 15, pp. 1579-1586, 1970.

[16] K. Yano and B. Y. Chen, "On concurrent vector fields of immersed manifolds," Kodai Mathematical Seminar Reports, vol. 23, pp. 343-350, 1971.

[17] B.-Y. Chen, "Classification of torqued vector fields and its applications to Ricci solitons," Kragujevac Journal of Mathematics, vol. 41, no. 2, pp. 239-250, 2017.

[18] K. Matsumoto, "On lorentzian paracontact manifolds," Bulletin of the Yamagata University. Natural science, vol. 12, pp. 151-156, 1989.

[19] I. Mihai and R. Rosca, On Lorentzian P-Sasakian Manifolds, Clssical Analysis, pp. 155-169, World Scientific Publishing, Signapore, 1992.

[20] I. Mihai, A. A. Shaikh, and U. C. De, "On Lorentzian paraSasakian manifolds, rendiconti del seminario matematico di Messina, serie II," IOSR Journal of Mathematics, vol. 9, no. 6, 1999.

[21] K. Matsumoto and I. Mihai, "On a certain transformation in a Lorentzian para Sasakian manifold," Tensor (N.S.), vol. 47, pp. 189-197, 1988.

[22] O. Bahadir and S. K. Chaubey, "Some result on LP-Sasakian manifolds with generalized symmetric metric connection," Honam Mathematical Journal, vol. 42, no. 3, pp. 461-476, 2020.

[23] U. C. De and A. K. Sengupta, "CR-submanifolds of a Lorentzian para-Sasakian manifold," Bulletin of the Malaysian Mathematical Sciences Society, vol. 23, pp. 99-106, 2000. 